
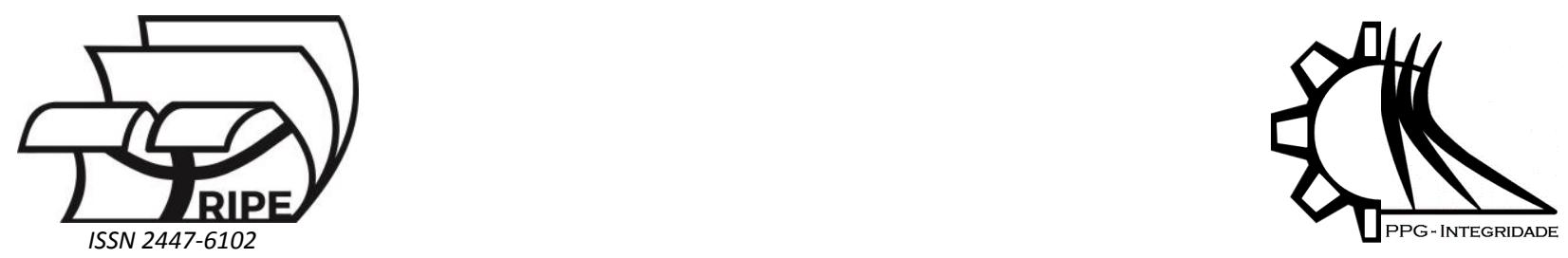

Article

\title{
Contribuição para estimativa do grau de saturação a partir das condições ambientais aplicada à análise de confiabilidade de estruturas de concreto sujeitas ao ataque por cloretos
}

\author{
Favretto, F. ${ }^{1}$, Magalhães, F.C. ${ }^{2}$, Real M.V. ${ }^{3}$ and Guimarães, A.T.C. ${ }^{4}$ \\ 1 Engenheiro Civil, Programa de Pós-graduação em Engenharia Oceânica, Universidade Federal do Rio Grande; \\ f2favretto@gmail.com \\ 2 Professor Doutor, Laboratório de Estruturas e Materiais de Construção Civil - LEMCC-IFRS-Campus Rio Grande, \\ fabio.magalhaes@riogrande.ifrs.edu.br \\ 3 Professor Doutor - Programa de Pós-graduação em Engenharia Oceânica Universidade Federal do Rio Grande, \\ mauroreal@furg.br \\ 4 Professor Doutor - Programa de Pós-graduação em Engenharia Oceânica Universidade Federal do Rio Grande, \\ atcg@vetorial.net
}

Received: 14/12/2018; Accepted: 05/01/2019; Published: 29/01/2019

\begin{abstract}
RESUMO: A durabilidade de elementos estruturais de concreto armado sujeitos a condições ambientais de severa agressividade apresenta-se como um dos principais problemas enfrentados na construção civil. Dentre os agentes de deterioração dessas estruturas destacam-se os íons cloretos, amplamente difundidos em ambiente marinho, que causam a corrosão de armaduras. A estimativa de vida útil de elementos de concreto armado surge como uma temática fundamental na avaliação da durabilidade das estruturas. Embora outros processos causem penetração de íons cloreto no concreto, é praticamente consenso que a difusão é o mecanismo predominante. Um modelo clássico de caracterização da penetração de cloretos é a 2a Lei de Fick. Guimarães (2000) demonstrou que o grau de saturação (GS) do concreto é um parâmetro que exerce forte influência sobre o coeficiente de difusão de cloretos (D). Embora tenham sido desenvolvidas metodologias de medição do GS, estas se apresentam onerosas em termos de tempo e recursos. $O$ objetivo deste trabalho é apresentar estimativas de GS com base nas condições ambientais a que o material é exposto e posteriormente analisar a influência deste parâmetro na avaliação da estimativa de vida de elementos estruturais através da confiabilidade. São utilizados dados meteorológicos e de medição do GS obtidas em duas regiões litorâneas: Rio Grande, Brasil e Santa Pola, Espanha. Obteve-se modelos de estimativa do GS a partir das variáveis ambientais de cada região e também de ambas em conjunto. Avaliou-se então a influência destes modelos na estimativa de vida útil através da probabilidade de falha da estrutura.
\end{abstract}

PALARAS CHAVE: Concreto. Cloretos. Grau de saturação. Durabilidade. Confiabilidade.

\section{Contribution to estimate of the saturation degree from environmental conditions applied to the reliability analysis of concrete structures subject to chloride attack}

ABSTRACT: The durability of structural elements of reinforced concrete subjected to environmental conditions of severe aggressiveness presents itself as one of the main problems faced in civil construction. Among the agents of deterioration of these structures stand out the chlorides ions, widely diffused in marine environment, that cause corrosion of steel reinforcement. The estimated useful life of reinforced concrete elements appears as a fundamental theme in the evaluation of the durability of the structures. Although other processes cause penetration of chloride ions into the concrete, it is practically a consensus that diffusion is the predominant mechanism. A classical model for characterizing chloride penetration is Fick's 2nd Law. Guimarães (2000) demonstrated that the saturation degree (GS) of the concrete is a parameter that exerts a strong influence on the diffusion coefficient of 
chlorides (D). Although GS measurement methodologies have been developed, they are costly in terms of time and resources. The objective of this work is present estimates of GS based on the environmental conditions to which the material is exposed and later analyze the influence of this parameter in the evaluation of the useful life of structural elements through the reliability. Meteorological and measurement data of GS obtained in two coastal regions are used: Rio Grande, Brazil and Santa Pola, Spain. Models of GS estimation were obtained from the environmental variables of each region and also from both together. The influence of these models on the estimation of useful life through the probability of failure of the structure was then evaluated.

KEYWORDS: Concrete. Chlorides. Saturation Degree. Durability. Reliability.

\section{Introdução}

Através da observação da deterioração precoce de estruturas de concreto armado, necessidade de maior competitividade no setor e do foco nos aspectos da sustentabilidade se fez necessário reavaliar e aperfeiçoar as técnicas e materiais utilizados nas estruturas de concreto, enfatizando-se os aspectos de projeto relativos a vida útil das construções. Dos problemas relativos a deterioração das estruturas de concreto verifica-se que grande parte se dá devido a corrosão das armaduras, problema geralmente associado a ação de agentes agressivos, em especial, à penetração de íons cloreto na massa de concreto, íons estes presentes em zonas marítimas, onde se enquadra o ambiente de estudo desta pesquisa. (CLIFTON, 1993; BICZÓK, 1972; ANDRADE, 1992; HELENE, 1993; DAL MOLIN, 1988; GJøRV et al., 1994; HELENE, 1986).

O processo de corrosão de estruturas de concreto armado, comumente é modelado em duas etapas: sendo a iniciação, correspondente ao período de ingresso dos íons cloreto até o fim da camada de cobrimento e consequente atingimento do Estado Limite de Serviço (ELS), e a propagação que se refere aos efeitos pós-despassivação do aço (corrosão) que gera fissuração do concreto e perda de seção da armadura. Em termos de tempo, o período de iniciação é consideravelmente maior que o de propagação, fato que justifica a importância dos estudos focados no período de iniciação (YU et al., 2017).

A maioria dos modelos de avaliação do processo de iniciação por cloretos, baseia-se em um modelo determinístico, por exemplo a solução da 2a Lei de Fick, e, através da estimativa de variabilidade das variáveis envolvidas, realizam uma análise em termos probabilísticos. Devido a aleatoriedade de diversas das variáveis envolvidas no processo, os modelos probabilísticos melhor retratam o período de iniciação.

Tendo-se em vista a importância do grau de saturação na correta avaliação da penetração de íons cloreto no concreto (GUIMARÃES e HELENE 2000; GUIMARÃES, 2009; MARTYS, 1999; MERCADO-MENDOZA et al., 2012) e da dificuldade técnica e econômica de se estimar esta propriedade, inviabilizando seu uso, busca-se facilitar o processo de determinação do GS através da avaliação dos parâmetros ambientais.

De posse de medições de GS de diferentes concretos expostos a atmosfera marinha em zona de névoa e de dados meteorológicos busca-se gerar modelos de estimativa do grau de saturação a partir das variáveis ambientais do local. Estas estimativas poderão ser utilizadas na solução do modelo de iniciação por cloretos para concretos de mesmas características dos estudados e em ambientes semelhantes aos pesquisados.

A durabilidade das estruturas é uma propriedade inerente a interação da estrutura com o ambiente em que se encontra inserida. Apesar dos efeitos da corrosão só serem percebidos após um avançado estágio de degradação, o marco que pode delimitar o fim da vida útil da estrutura é a despassivação da armadura (HELENE, 1993). Neste espectro de uma abordagem conservadora se torna fundamental estimar o tempo em que ocorre a despassivação das armaduras, viabilizando a adoção de medidas de prevenção e manutenção para um prolongamento da vida útil das estruturas (VERA et al., 2017).

Os principais agentes agressivos responsáveis pela corrosão de armaduras são o gás carbônico $\left(\mathrm{CO}_{2}\right)$, os sulfatos e os cloretos, amplamente presentes em ambientes marinhos e indústrias de fertilizantes. No presente trabalho serão desconsiderados os dois primeiros, sendo abordadas as características e consequências do ataque por cloretos.

A corrosão de armaduras devido ao ataque de íons cloreto é um dos problemas mais graves observados em estruturas de concreto armado. A película de passivação que envolve o aço logo após a hidratação do cimento pode ser destruída por estes íons, despassivando a armadura e dando início ao processo de corrosão na presença de água e oxigênio (NEVILLE, 1997).

Existem diversos mecanismos de transporte de íons e moléculas através da rede de poros do concreto. Os principais fenômenos conhecidos são a penetração por permeabilidade; a absorção capilar; a migração, o fluxo por 
convecção e a difusão. Apesar dos vários processos de transporte de íons muitas vezes ocorrerem simultaneamente, tem-se como consenso que o mecanismo da difusão é o predominante e é amplamente utilizado em modelos de predição da iniciação por íons cloreto (YU et al., 2017). A solução comumente utilizada para resolução do problema de difusão no concreto é a 2 a lei de Fick, com a qual é possível descrever para um determinado tempo a concentração de íons com a profundidade de penetração.

O coeficiente de difusão de cloretos é um importante parâmetro na estimativa precisa de vida útil das estruturas. Este coeficiente representa a difusibilidade de determinado concreto, que pode variar devido as propriedades dos materiais componentes do concreto e às características ambientais do local. A simplificação de cálculo geralmente adotada de se considerar D constante pode gerar muita imprecisão na estimativa de vida útil de estruturas, necessitando-se assim a utilização de parâmetros de correção deste coeficiente através dos principais fatores que influenciam em sua variação. Sendo que os fatores mais influentes sobre D são, dentre outros: o tipo de cimento; as adições; a relação água-cimento (a/c); a cura do concreto; a temperatura ambiente; e a umidade, relacionada através do GS do concreto.

O GS do concreto influencia diretamente o transporte de íons cloretos através da difusão na microestrutura de poros. O concreto apresenta uma rede de poros de diversos tamanhos, dos quais, os poros acima de um determinado diâmetro, chamado crítico, estão interligados, permitindo o transporte de fluidos no seu interior. Diversos autores como Martys, (1999); Guimarães, (2000); Guimarães e Helene, (2001); Climent et al. (2002); Nielsen e Geiker (2003); Guimarães (2005); Mercado-Mendoza et. al., (2012); Mercado-Mendoza et al. (2014); comprovaram a influência do grau de saturação do concreto no coeficiente de difusão de cloretos. Mais informações podem ser obtidas em seus trabalhos.

A variação do GS do concreto pode ser relativa a diversos fatores como: zona de inserção; características do concreto; posição da superfície exposta em relação a superfície de concretagem; condições de exposição no ambiente tais como sentido da superfície (horizontal ou vertical) e orientação (norte, sul, leste, oeste, para cima e para baixo). Para cada condição de exposição o GS do concreto pode ser influenciado por variáveis meteorológicas distintas. Peraça (2009) concluiu que para as condições dos testemunhos utilizados em sua pesquisa, as variáveis mais influentes na variação do GS foram a temperatura máxima (TM) e a umidade relativa (UR). Já Lopez (2011) em Santa Pola, Alicante, na Espanha, elencou as variáveis temperatura máxima, taxa de precipitação, horas de insolação e umidade relativa como mais influentes.

Estimar a confiabilidade ou consequente probabilidade de falha de determinada estrutura caracteriza-se como mensurar probabilisticamente o nível de segurança alcançado. Devido a aleatoriedade dos parâmetros envolvidos, os modelos determinísticos acabam não sendo precisos na estimativa do processo de deterioração por cloretos. De tal maneira, o uso de modelos probabilísticos torna-se fundamental para a avaliação dos processos que estabelecem a deterioração das estruturas de concreto armado. O presente trabalho busca utilizar a teoria da confiabilidade na avaliação da influência do GS, obtido através das condições ambientais, na estimativa de vida útil no ELS. O objetivo não é aprofundar-se em fundamentos teóricos de confiabilidade, bem como os métodos de solução e aplicação dos procedimentos estocásticos. Mais detalhes sobre estes podem ser obtidos em Melchers e Beck (2018), Andrade (2001), Magalhães (2018) e Haldar, e Mahadevan (2000).

As teorias de confiabilidade permitem calcular as incertezas e determinar a probabilidade de um sistema estrutural atingir um estado limite determinado. Os conceitos básicos de confiabilidade estão associados ao nível de confiança no qual uma estrutura apresenta um adequado desempenho em serviço. Os processos de projeto buscam garantir uma determinada probabilidade pré-ajustada de falha, ou no caso específico e esperado, de sucesso. (SANTIAGO, 2011; MAGALHÃES, 2014).

Como forma de avaliar a confiabilidade do problema para duas variáveis, capacidade resistente $(R)$ e solicitação $(S)$, define-se a função margem de segurança (M) do sistema estrutural como a diferença entre a resistência (R) da estrutura e a ação (S) a qual a estrutura é submetida, conforme a Eq. (1).

$$
M=R-S
$$

O índice de confiabilidade ( $\beta$ ) é definido pela Eq. (2), conforme observado pela Fig. 1:

$$
\beta=\frac{\mu_{M}}{\sigma_{M}}
$$

Onde:

$\mu_{M}$ é a média da margem de segurança;

$\sigma_{M}$ é o desvio padrão da margem de segurança; 


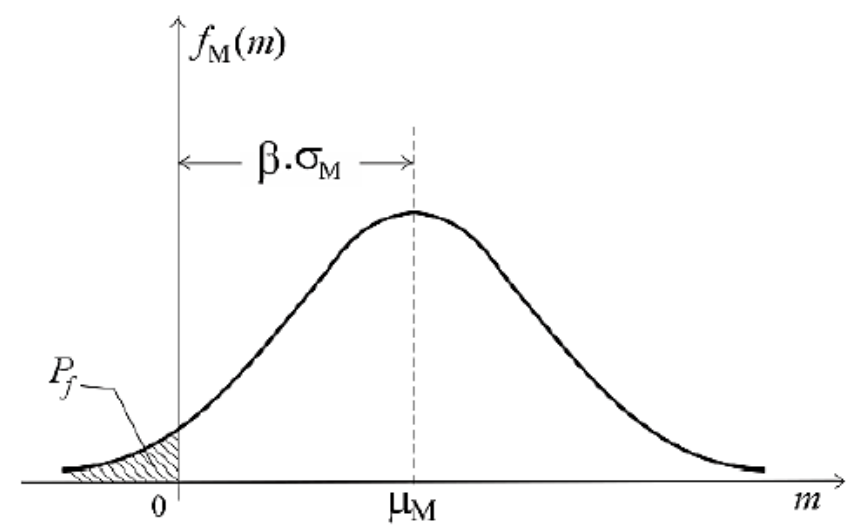

Figura 1. Função de densidade de probabilidades da margem de segurança (Fonte: Adaptado de Ang e Tang, 1984).

Tomando-se como Normal a distribuição de probabilidades acumulada ( $\Phi)$ da margem de segurança através da Eq. (3) tem-se:

$$
P_{f}=\Phi(-\beta)
$$

O método de Simulação de Monte Carlo (MCS) é baseado na geração de números aleatórios para variáveis com distribuição de probabilidade conhecida (ou assumida) permitindo simular o comportamento de determinado evento modelado através da função de falha $g(X)=0$. Esta metodologia consiste na realização de inúmeras simulações, onde o conjunto de resultados individuais obtidos permite a realização de uma análise estatística, possibilitando a determinação das características de distribuição de probabilidades das variáveis de resposta do sistema (MAGALHÃES, 2014).

Devido a dificuldade de recuperação das estruturas após iniciado o processo de corrosão do aço, diversos autores (YU et al., 2017; ANDRADE, 2001; BENTZ, 2003; GJØRV, 2015) adotam o início da corrosão como o atingimento do ELS. Magalhães (2018) relata que o processo de deterioração inicia-se quando os cloretos atingem um teor crítico $\left(C_{\text {Crit }}\right)$ no ponto analisado. Desta forma, a equação que representa a confiabilidade do elemento estrutural em termos de estado limite de serviço quanto da entrada de agentes agressivos é dada pela Eq. (4):

$$
g(x, t)=C_{\text {Crit }}-C(x, t)
$$

Sendo $C(x, t)$ a concentração de cloretos na armadura considerando-se um cobrimento $x$ e um tempo t. A Fig. 2 apresenta um resumo da metodologia proposta através de um fluxograma de trabalho. 


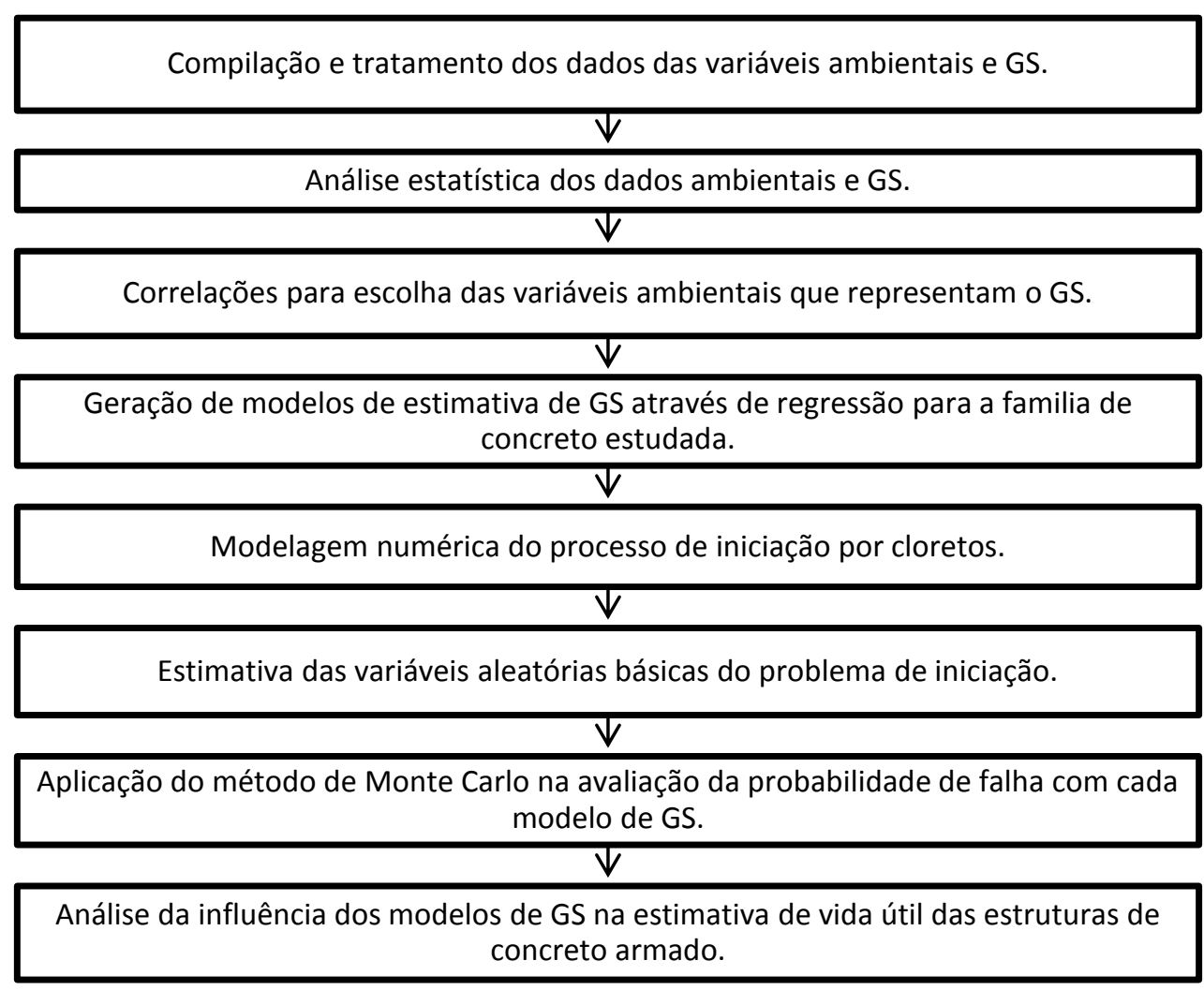

Figura 2. Fluxograma da metodologia proposta.

\section{Metodologia}

Visando a avaliação do comportamento do grau de saturação a partir da influência dos fatores ambientais e seu impacto na vida útil de serviço das estruturas, o presente trabalho utiliza dados experimentais obtidos em duas regiões distintas para microambiente de névoa. São adotados resultados do extremo sul do Brasil, na cidade de Rio Grande, Rio grande do Sul (PERAÇA, 2009) e de Santa Pola, Alicante, localizada no sudeste da Espanha (LOPEZ, 2011).

Para escolha das variáveis ambientais mais influentes sobre o GS realizou-se correlação estatística das mesmas com o GS medido de cada família de concreto, que corresponde ao CP 1 e 2 (PERAÇA, 2009) e CP 30 (LOPEZ, 2011). Através do parâmetro de correlação ( $r$ ) elencou-se as variáveis mais influentes para compor o modelo.

Através da regressão linear gerou-se modelos de estimativa de GS para 3 casos distintos: dados oriundos do Brasil; dados oriundos da Espanha; e ambos conjuntos de dados agrupados (Brasil e Espanha).

A solução da 2a - Lei de Fick foi utilizada como modelo básico para simular o comportamento da entrada de agentes agressivos no concreto. $O$ critério de falha adotado para a avaliação da confiabilidade é o atingimento de um teor crítico de cloretos na profundidade de cobrimento correspondendo ao ELS, que pode ser expresso através da Eq. (4):

Adotou-se coeficientes de correção de $D_{0}$ propostos por Guimarães (2000), sendo o modelo matematicamente descrito através da Eq. (5).

$$
C_{(x, t)}=C_{S} \cdot \operatorname{erfc}\left(\frac{x}{2 \sqrt{R_{C} \cdot R_{G S} \cdot R_{T} \cdot R_{S C} \cdot D_{0} \cdot t}}\right)
$$

Onde:

$C_{S}$ é a concentração superfial de cloretos em função do tempo;

erfc( ) é a função complementar erro de Gauss;

$R_{C}$ é o coeficiente de correção devido à influência do cimento utilizado;

$R_{G S}$ é o coeficiente de correção devido à influência do GS do concreto;

$\mathrm{R}_{\mathrm{T}}$ é o coeficiente de correção devido à influência da temperatura ambiente;

$\mathrm{R}_{\mathrm{SC}}$ é o coeficiente de correção devido à influência da superfície exposta em relação à concretagem;

$D_{0}$ é o coeficiente de difusão de referência do concreto. 
Para realização das simulações o modelo foi programado em linguagem Octave, onde desenvolveu-se 5 rotinas para simulação do problema proposto, as rotinas programadas variam a consideração da influência do GS na penetração de cloretos e consequente probabilidade de falha no atingimento do ELS. Desenvolveu-se uma rotina para cada modelo de GS apresentado, uma desconsiderando o coeficiente de correção Rgs, ou seja, considerando o grau de saturação igual a 100\% e também uma para Rgs=0,205 (GUIMARÃES, 2005) com coeficiente de variação igual a 0,05.

Para verificação do processo de simulação foram utilizados dados experimentais de elementos estruturais de concreto armado em ambiente de severa agressividade apresentados por Guimarães (2000). Tratam-se de resultados obtidos da estrutura do cais Terminal de Containers (TECON) do superporto da cidade de Rio Grande - RS (BRETANHA, 2004; GUIMARÃES, 2005). Os dados utilizados são do elemento de concreto situado em zona totalmente de névoa denominado PS.

Os valores médios das variáveis utilizadas nas avaliações realizadas, bem como suas distribuições de probabilidades e dispersões, estão apresentados na Tab. 1.

Tabela 1. Parâmetros e distribuição de probabilidades das variáveis utilizadas na avaliação da confiabilidade.

\begin{tabular}{|c|c|c|c|c|c|}
\hline Variável & Unidade & Média & $\begin{array}{l}\text { Coef. de variação } \\
\text { (V) }\end{array}$ & $\begin{array}{l}\text { Distribuição } \\
\text { estatística }\end{array}$ & Referência \\
\hline$C_{S}$ & - & 2,46 & 0,20 & LN & Yu et al. (2017) \\
\hline \multirow{2}{*}{$C_{c r i t}$} & \multirow{2}{*}{$\%$} & \multirow{2}{*}{0,40} & \multirow{2}{*}{0,15} & \multirow{2}{*}{$\mathrm{N}$} & Helene (1993) \\
\hline & & & & & Yu et al. (2017) \\
\hline \multirow{3}{*}{$\mathrm{R}_{\mathrm{GS}}$} & - & 0,205 & 0,05 & LN & Guimarães (2005)* \\
\hline & - & 1,00 & 0,00 & - & - \\
\hline & - & \multicolumn{3}{|c|}{ Conforme modelos de GS } & Autor \\
\hline $\mathrm{R}_{\mathrm{T}}$ & - & 0,81 & 0,20 & $\mathrm{~N}$ & Bretanha (2004)* \\
\hline $\mathrm{R}_{\mathrm{SC}}$ & - & 0,74 & 0,20 & LN & Guimarães (2000)* \\
\hline $\mathrm{R}_{\mathrm{C}}$ & - & 0,64 & 0,00 & - & - \\
\hline $\mathrm{D}_{\mathrm{a}}$ & $\mathrm{cm}^{2} /$ ano & 104 & 0,10 & $\mathrm{~N}$ & Bentz (2003) \\
\hline$x$ & $\mathrm{~cm}$ & $x$ MÉDIO & 0,10 & $\mathrm{~N}$ & Magalhães (2014) \\
\hline
\end{tabular}

N - Normal; LN - LogNormal; *obtido através dos dados apresentados pelos autores.

Para avaliação da confiabilidade, através do modelo proposto, utilizou-se o método de Simulação de Monte Carlo (MCS), analisando-se a probabilidade de falha do sistema através da Eq. (6), realizando-se 1.000.000 de simulações.

$$
P_{f}=\frac{N f}{n}
$$

Onde:

$N f$ é número de falhas;

$n$ é o número de simulações;

$P_{f}$ é a probabilidade de falha. 


\section{Resultados e discussões}

Analisando-se os resultados do coeficiente de correlação ( $r$ ) de cada variável ambiental com o corpo de prova (CP) que melhor representa o ponto de estudo PS, elencou-se as variáveis ambientais mais influentes sobre o GS de cada ambiente e também de ambos os ambientes em conjunto, obtendo-se os resultados apresentados na Tab. 2.

Tabela 2. Resultado das correlações das variáveis com GS.

\begin{tabular}{|c|c|c|c|c|}
\hline \multicolumn{5}{|c|}{ Correlação (r) } \\
\hline VARIÁVEIS & $\begin{array}{c}\text { Temperatura de ar } \\
\text { úmido (TAU) }\end{array}$ & $\begin{array}{c}\text { Temperatura do } \\
\text { ar seco (TAS) }\end{array}$ & $\begin{array}{l}\text { Temperatura } \\
\text { Máxima (TM) }\end{array}$ & $\begin{array}{l}\text { Temperatura } \\
\text { Mínima (TMíN) }\end{array}$ \\
\hline $\begin{array}{l}\text { GS CPS } 1 \text { e } 2 \text { Rio Grande } \\
\text { (médias sazonais) }\end{array}$ & $-0,8472$ & $-0,8646$ & $-0,8776$ & $-0,8495$ \\
\hline VARIÁVEIS & $\begin{array}{l}\text { Temperatura } \\
\text { Máxima (TM) }\end{array}$ & $\begin{array}{l}\text { Umidade Relativa } \\
\text { (UR) }\end{array}$ & $\begin{array}{c}\text { Taxa de } \\
\text { Precipitação (TP) }\end{array}$ & $\begin{array}{c}\text { Horas de Insolação } \\
\text { (HI) }\end{array}$ \\
\hline $\begin{array}{l}\text { GS VCP1 (30) Santa Pola } \\
\text { (médias semanais) }\end{array}$ & $-0,3216$ & 0,6233 & 0,2837 & $-0,3319$ \\
\hline VARIÁVEIS & $\begin{array}{l}\text { Temperatura } \\
\text { Máxima (TM) }\end{array}$ & $\begin{array}{l}\text { Temperatura } \\
\text { Mínima (TMíN) }\end{array}$ & $\begin{array}{c}\text { Umidade } \\
\text { Relativa (UR) }\end{array}$ & $\begin{array}{c}\text { Taxa de } \\
\text { Precipitação (TP) }\end{array}$ \\
\hline $\begin{array}{l}\text { GS traço } 1 \text { (1; } 2 \text { e } 30 \text { ) Rio } \\
\text { Grande e Santa Pola } \\
\text { (médias semanais) }\end{array}$ & $-0,4496$ & $-0,3073$ & 0,6410 & 0,3544 \\
\hline
\end{tabular}

De posse das duas variáveis mais influentes na correlação de cada arranjo gerou-se, através da regressão linear múltipla, modelos de estimativa do GS da família de concreto do traço 1. Os modelos obtidos em cada caso bem como o coeficiente de determinação $\mathrm{R}^{2}$, que expressa a precisão do modelo obtido, são apresentados na Tab. 3.

Tabela 3. Modelos de estimativa de GS e coeficiente de determinação.

\begin{tabular}{c|r|c}
\hline MODELO & EQUAÇÃO & $\mathbf{R}^{\mathbf{2}}$ \\
\hline GS CPS 1 e 2 Rio Grande & $G S=80,908-1,343 \times T M$ & \\
(médias sazonais) & $+0,542 \times T A S$ & 0,774 \\
\hline GS VCP1 (30) Santa Pola & $G S=14,794-0,501 \times H I+$ & \\
(médias semanais) & $0,582 \times U R$ & 0,419 \\
\hline
\end{tabular}




\begin{tabular}{c|r|r}
\hline GS traço 1 (1; 2 e 30) Rio & $G S=36,032-0,386 \times T M$ & \\
Grande e Santa Pola & $+0,397 \times U R$ & 0,513 \\
(médias semanais) & & \\
\hline
\end{tabular}

Obteve-se para cada variável dos modelos o tipo de distribuição de probabilidade e seus parâmetros, como média $(\mu)$, desvio padrão $(\sigma)$ e coeficiente de correlação $(\rho)$ das duas variáveis ambientais de cada modelo. Os resultados obtidos são apresentados na Tab. 4.

Tabela 4. Parâmetros estatísticos das variáveis de entrada.

\begin{tabular}{|c|c|c|c|c|}
\hline VARIÁVEL & DIST. EST. & $\mu$ & $\sigma$ & $\rho$ \\
\hline TAS média sazonal RG & Normal & 18,623 & 3,626 & \multirow{2}{*}{0,9934} \\
\hline TM média sazonal RG & Normal & 23,589 & 3,82 & \\
\hline UR média semanal SP & Normal & 69,457 & 6,196 & \multirow{2}{*}{$-0,26$} \\
\hline HI média semanal SP & Normal & 6,12 & 1,964 & \\
\hline TM média semanal SP+RG & Normal & 23,031 & 4,606 & \multirow{2}{*}{$-0,215$} \\
\hline UR média semanal SP+RG & Normal & 78,621 & 7,805 & \\
\hline
\end{tabular}

Para evitar erros dos valores extremos obtidos nos modelos, causados pela geração de números aleatórios, optou-se por truncar o valor de GS obtido em cada modelo em 5\% abaixo e acima do menor e do maior valor do GS medido, respectivamente, para cada arranjo. O resumo dos valores adotados para truncagem estão na Tab. 5 .

Tabela 5. Valores de truncagem de GS.

\begin{tabular}{c|c|c}
\hline MODELO & GS Máximo & GS Mínimo \\
\hline Sazonais) & 70,01 & 51,53 \\
semanais) 1 e $\mathbf{2}$ Rio Grande (médias & 71,55 & 40,84 \\
\hline GS VCP1 (30) Santa Pola (médias & & \\
\hline GS traço 1 (1; 2 e 30) Rio Grande e & 73,26 & 40,84 \\
Santa Pola (médias semanais) & & \\
\hline
\end{tabular}

Para geração do valor do fator de correção Rgs adotou-se modelo obtido através da regressão linear simples apresentado na Eq. (7). Os dados utilizados foram obtidos por Guimarães (2005), o modelo proposto é limitado a valores de GS entre 40 a 90\%, sendo satisfatório para o GS obtido nos modelos de estimativa propostos.

$$
R g s=-0,2789+0,006528 * G S \quad p / \quad 40 \%<G S<90 \%
$$


A convergência estatística da média $(\mu)$ e desvio padrão $(\sigma)$ da concentração de cloretos pode ser verificada através dos resultados do modelo desconsiderando o Rgs, para um tempo de 50 anos e diversas profundidades (Fig. 3), os demais modelos também apresentaram convergência estatística satisfatória para o número de simulações utilizado. Apesar de haver convergência do modelo para número de simulações em torno de 5.000 optou-se por utilizar 1.000.000 de simulações no processo para minimizar os erros.

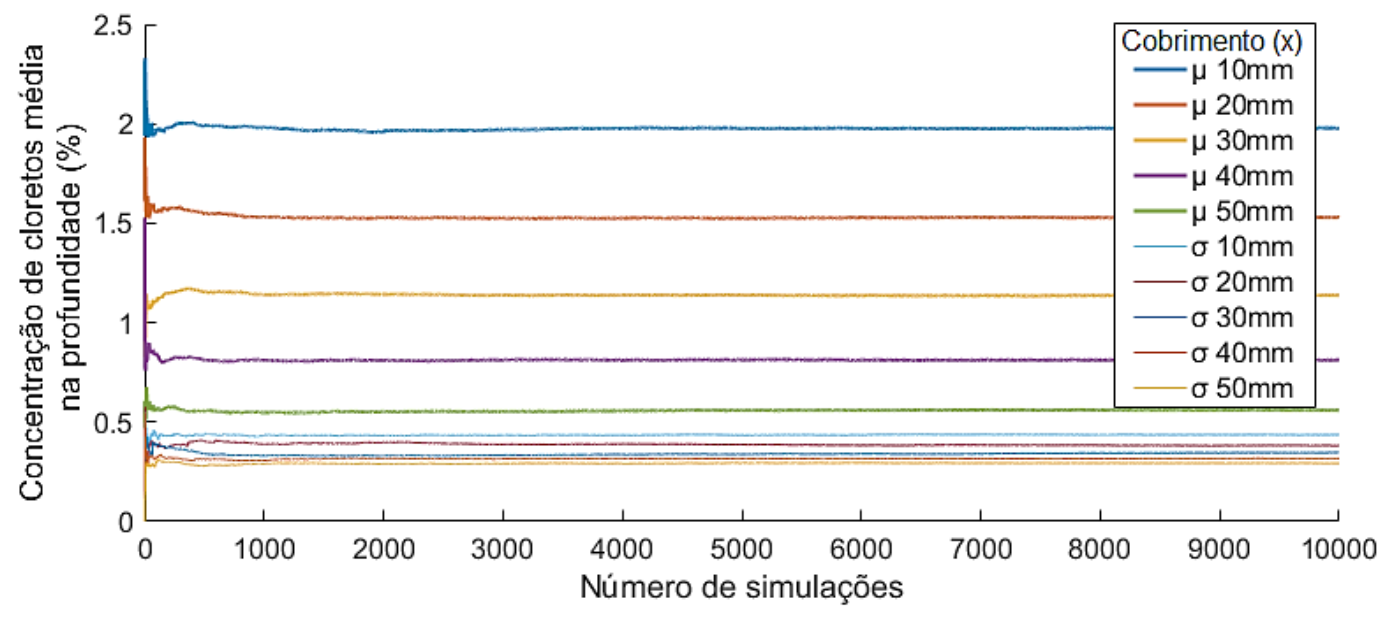

Figura .3. Convergência da média e desvio padrão da concentração de cloretos para diversos cobrimentos.

A probabilidade de falha de cada modelo em função do cobrimento para uma idade de 50 anos é apresentada na Fig. 4.

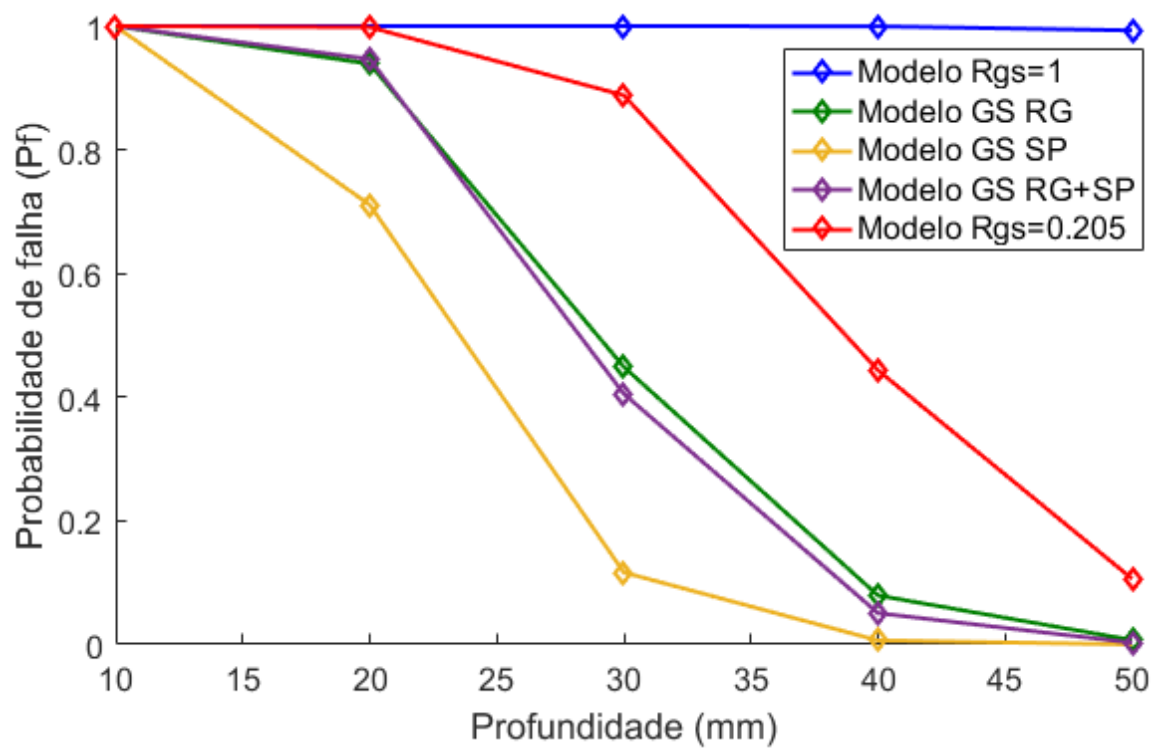

Figura 4. Probabilidade de falha dos modelos pela profundidade.

Para avaliação da influência do uso dos diferentes modelos de estimativa do GS e seu impacto sobre a concentração de cloretos na profundidade, optou-se por avaliar a probabilidade de falha da estrutura. Para tal, utilizou-se os modelos de GS propostos, alimentados pela geração de números aleatórios das variáveis ambientais apresentadas na Tab. 4, com suas respectivas distribuições de probabilidade. Os demais parâmetros foram apresentados na Tab. 1. Para efeito de comparação também se apresenta um modelo considerando Rgs=1(GS=100\%) e Rgs $=0,205$. 
Pode-se observar que para a função de falha adotada há uma grande diferença entre os modelos de GS utilizados, salienta-se que apenas se apresenta a variação dos resultados da probabilidade de falha em função do modelo de GS ou Rgs adotado, cabendo a definição de qual o melhor modelo a ser utilizado pela comparação com dados experimentais consistentes. $\mathrm{O}$ modelo considerando $\mathrm{Rgs}=1$ apresentou o resultado mais discrepante, com comportamento diferente dos demais, tal fato pode justificar a grande relevância da consideração do coeficiente de correção Rgs na previsão de vida útil, pois considerar o concreto totalmente saturado leva a uma subestimação da mesma.

\section{Conclusões}

Do estudo da correlação obtiveram-se as variáveis ambientais mais influentes que foram TM e TAS para Rio Grande, UR e HI para Santa Pola e TM e UR para os ambientes em conjunto. É possível constatar que há uma diferença no comportamento do GS perante as variáveis ambientais, haja visto que os parâmetros dos modelos foram diferentes, porém cabe ressaltar que as medições de GS de Santa Pola não englobaram as 4 estações do ano o que pode afetar a correlação das variáveis. Também existe algumas diferenças climáticas entre os ambientes, propiciando que o GS médio em Santa Pola seja de $51,44 \%$ e em Rio Grande $59,45 \%$.

Analisando-se os resultados da probabilidade de falha obtidos nas simulações, constatou-se a importância da consideração do GS nos modelos de vida útil. A não consideração do coeficiente de correção devido à influência do GS leva a obter-se elevadas probabilidades de falha, o que pode não representar a realidade, pois subestima a vida útil da estrutura. Também há diferenças significativas nos resultados obtidos dos demais modelos, ficando esta análise sujeita apenas a comprovar a influência deste parâmetro na estimativa de vida útil de estruturas de concreto armado. A escolha de qual modelo de GS deve ser utilizado nas estimativas de vida útil passa pela validação do mesmo, com base em dados experimentais consistentes de estruturas com características semelhantes.

Agradecimentos: O presente trabalho foi realizado com apoio da Coordenação de Aperfeiçoamento de Pessoal de Nível Superior Brasil (CAPES) - Código de Financiamento 001.

\section{Referências}

1. Andrade, J.J.O. Contribuição à previsão da vida útil das estruturas de concreto armado atacadas pela corrosão de armaduras: iniciação por cloretos. Tese (Doutorado em Engenharia Civil) - Universidade Federal do Rio Grande do Sul, Porto Alegre - RS, 2001.

2. Andrade, M.C. "Manual para diagnóstico de obras deterioradas por corrosão de armaduras." Tradução: Antônio Carmona e Paulo Helene. Pini, SP, 1992.

3. Ang, A. H-S; tang, W. H. Probability concepts in engineering planning and design. Volume II: Decision, risk, and reliability. New York, John Wiley \& Sons, 1984.

4. Bentz, E. C., Probabilistic modeling of service life for structures subjected to chlorides. ACl Materials Journal, v. 100, Issue 5, p. 391-397, 2003.

5. Biczók, I. “Corrosion y proteccion del hormigon." Tradução: Emilio J. Dócon Asensi. Bilbao, Ediciones Urmo, 1972.

6. Bretanha, S.S.F. "Variação do Grau de Saturação do Concreto em Ambiente Marítimo." Rio Grande, RS, dezembro de 2004. 56-66. Dissertação (Mestrado em Engenharia Oceânica). FURG - Universidade Federal do Rio Grande.

7. Clifton, James R. Predicting the Service Life of Concrete. ACI Materials Journal, v. 90, n. 6, p. 611-16, 1993.

8. Climent, M. A.; Vera, G.; López, J. F.; Viqueira, E.; Andrade, C. A Test Method for Measuring Chloride Diffusion Coefficients Through Nonsaturated Concrete Part I: The Instantaneous Plane Source Diffusion Case. Cement and concrete Research, v 32, 2002, p. 1113-1123.

9. Dal Molin, D.C.C. "Fissuras em estruturas de concreto armado: análise das manifestações típicas e levantamento de casos ocorridos no Estado do Rio Grande do Sul." Porto Alegre, RS, 1988. Dissertação (Mestrado em Engenharia Civil). UFRGS - Universidade Federal do Rio Grande do Sul.

10. Gjørv, O.E., K. Tan, e M. Zhang. "Diffusivity of chlorides from seawater into

high-strength lightweight concrete." ACI Materials Journal. ACI, USA, setembro/outubro de 1994. 447-452

11. Gjørv, O. E. Projeto da durabilidade de estruturas de concreto em ambientes de severa agressividade. Trad. Leda Maria Marques Dias Beck, São Paulo, Oficina de Textos, 2015 
12. Guimarães, A. T. C. Vida útil de estruturas de concreto armado em ambientes marítimos. Tese (Doutorado), Universidade de São Paulo - PCC/USP, Engenharia Civil, São Paulo, 2000.

13. Guimarães, A. T. C.; HELENE, P. R. L. Influência do teor de umidade sobre a difusividade de cloretos na pasta de cimento. Relatório FAPESP processo 99/10909-7. São Paulo, 2001.

14. Guimarães, André T.C. "Grau de Saturação: Sua Variação com o Tipo de Concreto e sua Infuência na Difusão de íons Cloreto." São Paulo, SP, abril de 2005. Monografia (Pós-Doutorado em Engenharia Civil). USP - Escola Politécnica da Universidade de São Paulo.

15. Guimarães, André T.C. e HELENE, Paulo R.L. "Modelos para Previsão de Vida Útil em Ambiente Marítimo." In: 42ํㅡㄹ Congresso Brasileiro do Concreto. Fortaleza, CE: IBRACON, 2000-B. 13-18.

16. Haldar, A. Mahadevan, S. Reliability Assessment Using Stochastic Finite Element Analysis, John Wiley \& Sons, 2000.

17. Helene, P. R. L. Contribuição ao estudo da corrosão em armaduras de concreto armado. Tese (Livre Docente), Escola Politécnica da Universidade de São Paulo, Departamento de Engenharia Civil, São Paulo, 1993.

18. Helene, Paulo R.L. "Corrosão em Armaduras para Concreto Armado." PINI / IPT, SÃO PAULO, 1986. P.45.

19. Lopez, E. F. V. "Estudio de la influencia de las varibles meterologicas em el grado de saturacion en hormigones expuesto a un ambiente marino atmosferico mediterraneo". Trabajo fin de Master. Universidad de Alicante, Master en Ingenieria de los Materiales, Agua y Terreno. Universidad de Alicante, Alicante, Julio, 2011.

20. Magalhães, F. C. A problemática dos concretos não conformes e sua influência na confiabilidade de pilares de concreto armado. 2014. Tese (Doutorado em Engenharia Civil) - Programa de Pós-Graduação em Engenharia Civil, UFRGS, Porto Alegre-RS.

21. Magalhães, F. C., Previsão de defesa 2018. Análise probabilística de elementos estruturais sujeitos ao ataque de cloretos em ambiente de severa agressividade. Tese de Doutorado em Engenharia Civil: Construção e Infraestrutura, Programa de Pós-Graduação em Engenharia Civil: Construção e Infraestrutura, Universidade Federal do Rio Grande do Sul.

22. Martys, N. S. Diffusion in partially-saturated porous materials. Materials and Structures, v. 32, p. 555-562, October. 1999.

23. Melchers, R. E.; Beck, A. T. Structural Realiability Analysis and Prediction. Wiley, , 2018.

24. Mercado-Mendoza, H., Lorente, S., Bourbon, X. The Diffusion Coefficient of Ionic Species Through Unsaturated Materials. Transport in Porous Media. v.96, Issue 3, p. 469-481, November, 2012.

25. Mercado-Mendoza, H., Lorente, S., Bourbon, X. Ionic aqueous diffusion through unsaturated cementitious materials - A comparative study. Construction and Building Materials. v. 51, p. 1-8, 2014.

26. Neville, A. Propriedades do concreto, 2o Edição, São Paulo, Pini, 1997.

27. Nielsen, E. P., Geiker, M. R., Chloride diffusion in partially satured cementitions material. Cement and Concrete Research, v. 33, p. 133-138, 2003.

28. Peraça, M. G. T. Modelos para estimativa do grau de saturação do concreto mediante variáveis ambientais que influenciam na sua variação. Dissertação de Mestrado, FURG, Rio Grande, 2009.

29. Santiago, W. C. Estudo da (Não-)Conformidade de Concretos Produzidos no Brasil e sua Influência na Confiabilidade Estrutural. Dissertação de Mestrado. USP. São Carlos SP, 2011.

30. Vera, G.; Antón, C.; López, M.P.; Climent. M.A. Depassivation time estimation in reinforced concrete structures exposed to chloride ingress: A probabilistic approach. Cement and Concrete Composites, v.79 p.21-33, May 2017.

31. Yu, B.; Ning, C.; Li, B. Probabilistic durability assessment of concrete structures in marine environments: Reliability and sensitivity analysis. China Ocean Engineering, v.31 p.63-73, March 2017. 